\title{
Misophonia: a scoping review of research
}

Iskra Potgieter ${ }^{1,2}$, Carol MacDonald ${ }^{3}$, Lucy Partridge ${ }^{4}$, Rilana Cima ${ }^{5}$, Jacqueline Sheldrake ${ }^{6}$, Derek J Hoare ${ }^{1,2}$

${ }^{1}$ National Institute for Health Research (NIHR) Nottingham Biomedical Research Centre, Ropewalk House, 113 The Ropewalk, Nottingham, NG1 5DU, University of Nottingham, Nottingham, UK

${ }^{2}$ Otology and Hearing Group, Division of Clinical Neuroscience, School of Medicine, University of Nottingham, NG7 2UH, UK

${ }^{3}$ Department of Psychology, University of Stirling, Stirling, FK7 7LU, UK

${ }^{4}$ Nuffield Hearing \& Speech Centre, Royal National Throat Nose and Ear Hospital, 330 Gray's Inn Rd, London, WC1X 8DA, UK

${ }^{5}$ Maastricht Univerrsity, Dept of Clinical Psychological Science, Universiteitssingel 40, P.O. Box 616 |6200 MD Maastricht

${ }^{6}$ The Tinnitus and Hyperacusis Centre, 32 Devonshire Place, London W1G 6JL

Contact address: Derek J Hoare, National Institute for Health Research (NIHR) Nottingham Biomedical Research Centre, Ropewalk House, 113 The Ropewalk, Nottingham, NG1 5DU, UK.

Short Title: Current position on misophonia 


\section{Abstract}

Objective: To scope the literature describing misophonia populations, management, and research opportunities.

Method: Literature searches for research studies describing patients diagnosed with misophonia, defining a patient profile, or outlining, developing, or testing an intervention for. A data extraction for was developed and piloted before data from each article were independently charted by two researchers. Researchers then agreed a final dataset for each article.

Results: Thirty-one records were included. The misophonia population was described in terms of onset age, triggers, reaction, coping strategies, and comorbid conditions. We identified nine outcome measures. Case studies on treatments included Cognitive Behavioural Therapy, Counterconditioning, Mindfulness and Acceptance, Dialectical Behavioural Therapy and pharmaceuticals. Future research priorities identified included clarifying the phenomenology and prevalence of misophonia, and randomized controlled trials of treatments.

Conclusion: Misophonia is under researched but there are strong foundations for future research to finalize diagnostic criteria, validate outcome measures, and trial treatments.

Key words: diagnosis, outcome measures, research priorities, psychotherapy 


\section{Introduction}

Misophonia, literally meaning hatred of sound, was first referred to in research literature in 2002 to describe a consistent set of symptoms and has since been increasingly encountered by clinicians (Jastreboff \& Jastreboff, 2002; 2003; Bernstein, Angell \& Dehle 2013; McFerran 2016; Kumar et al., 2017).The initial definition of misophonia was 'an abnormally strong reaction occurring to a sound with a specific pattern and/or meaning to an individual' (Jastreboff \& Jastreboff, 2014). Typically, patients suffer a strong negative emotional and autonomic 'fight or flight' reaction triggered by specific sound stimuli termed 'trigger sounds' (Edelstein, Brang, Rouw, \& Ramachandran 2013; Jastreboff \& Jastreboff, 2013; Schröder, Vulnik \& Denys 2013b; Wu, Lewin, Murphy \& Storch 2014). Although variations exist, most commonly the patient experiences a disproportionate level of hate, anger, rage, and disgust towards a person producing sounds associated with eating (e.g. chewing, swallowing) or breathing (Edelstein et al., 2013). The symptoms usually occur irrespective of sound spectrum and intensity and are exacerbated within a familar context (Edelstein et al., 2013). An important exception is self-produced similar sounds which have the same spectrum and intensity but do not usually evoke these abnormal reactions. Disturbed by the inappropriateness of his or her reaction, the patient engages in maladaptive coping mechanisms such as avoiding social situations in which the trigger-sounds might occur. Ultimately this results in interpersonal and occupational impairment, anxiety, and obsessive compulsions. Patients with severe misophonia suffer from the intrusive and unwanted preoccupation with trigger-stimuli, as well as compulsive stress-reducing and avoidance behaviours (Cavanna \& Seri, 2015; Webber \& Storch, 2015) which are typically seen in patients with obsessive compulsive disorder (OCD) (Bruxner, 2016). Moreover, these reactions are in some cases equally evoked by repetitive visual person-related triggers (e.g. leg-rocking or watching somebody chew) termed misokinesia, suggesting the disorder 
expands beyond merely audiological symptoms, and confirming a strong likeness to OCD as well as Tourette syndrome. Other less frequent comorbid conditions include mood disorder, panic disorder, attention deficit hyperactivity disorder, hypochondria, trichotillomania, and skin picking. (Schröder et al. 2013b; Taylor, Conelea, McKay, Crowe \& Abramowitz 2014; Ferreira, Harrison \& Fontenelle 2013; Hazen et al., 2008; Johnson et al., 2013; McGuire, Wu \& Storch 2015; Taylor et al., 2014; Webber, Johnson \& Storch 2014).

Misophonia has, of yet, no official diagnostic formulation within the Diagnostic and Statistical Manual of Mental Disorders, Fifth Edition (DSM-V) or the International Statistical Classification of Diseases and Related Health Problems, 10th revision (ICD-10) (Kumar et al., 2017). Considering the parallels to OCD, it has been proposed that misophonia can be categorized within the obsessive compulsive spectrum of disorders in the DSM-V (Schröder 2013b). On the other hand, as it borders both psychiatry and audiology, misophonia is also considered to be categorizable with disorders in audiology including tinnitus (perception of constant internally-perceived abstract sounds), hyperacusis (discomfort/pain experienced due to everyday sounds of a normal or louder intensity), phonophobia (extreme fear towards specific trigger sounds). Misophonia is distinct from these disorders although there is some overlap in symptoms and an individual may present with more than one condition (Jastreboff 2000; Jastreboff and Jastreboff 2003; Fackrell et al., 2017; Schwartz, Leyendecker, \& Conlon 2011).

Currently, little is known about the aetiology of misophonia (Jastreboff and Jastreboff 2014). Propositions of the psychiatric nature of misophonia (Ferreira et al., 2013) are founded on the basis that it is not an auditory disorder caused by a neurological dysfunction. This is evidenced as misophonia patients typically have normal hearing thresholds (Schröder et al., 2013b). Instead, misophonia is associated with an increased number of connections, or 
strength of connections, between the limbic and sympathetic nervous systems which can cause abnormal processing of sound stimuli (Moller, 2011; Jastreboff \& Hazell, 2008; Jastreboff \& Jastreboff, 2013). Case studies also suggest a hereditary component in misophonia (Cavanna 2014).

Treatments for misophonia include Tinnitus Retraining Therapy and sound therapy (Jastreboff and Jastreboff 2013, Formby, Sherlock \& Gold 2003) and Cognitive Behavioural Therapy (CBT) (Schröder et al., 2017; Bernstein et al., 2013; McGuire et al., 2015; Reid, Guzick, Gernand \& Olsen 2016). Other less documented cognitive treatments for misophonia include Counterconditioning (Dozier 2015a), applied relaxation (Martz 2013), and the intake of serotonergic or anti-dopaminergic agents, which have been shown to have some efficacy on misophonia when it is within an OCD diagnosis (Neal \& Cavanna 2013; Eddy \& Cavanna 2013).

\section{Rationale for the review}

The research literature has noted a steady increase in the number of misophonia cases over the last decade (Wu et al., 2014; Schwartz et al., 2011; Hadjipavlou, Baer, Lau \& Howard 2008). Consequently, there has also been a rise in the number of relevant research studies (Duddy \& Oeding 2014), gradually compiling an evidence base for this new and independent condition. A point has now been reached where the literature on misophonia needs to be catalogued purposefully to provide an account of the current research position and guide future research priorities. This review on misophonia is the first to apply a scoping review methodology to the subject.

\section{Aims}


This scoping review aimed to collate the research evidence to date on the definition, patient profile, assessment, and treatment of misophonia, such that research gaps and opportunities could be identified. Specifically, our objectives relate to (1) how misophonia and the populations it affects are described in research literature, (2) what research has been conducted to date on the assessment and treatment of misophonia, and (3) in both cases, what are priority research questions.

\section{Methods}

This review is reported according to the PRISMA checklist extension for scoping reviews (PRISMA-ScR; Tricco et al., 2018). No protocol for the review was published. A methodological framework for scoping reviews was followed (Arksey \& O’Malley 2005, Levac, Colquhoun, \& O’Brien 2010; Peters et al., 2015; Khalil et al., 2016) involving six stages whereby (1) the purpose and research questions were defined, (2) relevant studies were identified using a three-step literature search in order to balance the breadth and comprehensiveness, (3) studies were selected using an iterative team approach to study selection and data extraction, (4) the data were charted incorporating qualitative thematic analysis where necessary, (5) the results were collated, summarized and reported, including the implications for policy, practice or research, and (6) the findings were reviewed by clinical experts in the field who did not take part in stages 1-5.

\section{Eligibility criteria}

Records were included if they (1) reported research studies (including studies of aetiology, symptomatology/patient profiles, prevalence, incidence, cohort and case control studies) and focused on describing the population of patients diagnosed with misophonia, or (2) reported case studies, case series, cohort, feasibility or pilot studies where the purpose was to define a 
patient profile or outline or develop an intervention for patients with a primary complaint of misophonia, or (3) reported randomized controlled trials (RCTs), non-RCT, case or cohort studies or case series where the effectiveness of an intervention for misophonia was tested and misophonia was the primary complaint or part of a symptom set.

Records were excluded if they were not peer reviewed, were reviews (our searches confirm there are no previous related scoping or systematic reviews), were personal/expert opinions and editorials, manuals or guidelines, patents, not available in English, or were reporting animal studies.

\section{Information sources and search strategy}

Initial searches were conducted in July 2016. Searches were conducted in Embase, PsychINFO, PubMed CENTRAL, Scopus, Web of Science, Cochrane CENTRAL, Cochrane Ear Nose and Throat Disorders Group Trials Register, Clinical Trials.gov, the International Standard Randomized Controlled Trial Number registry (ISRCTN), the International Clinical Trials Registry Platform (ICTRP), Cumulative Index to Nursing and Allied Health Literature (CINAHL PLUS) and Google Scholar. We applied a specific search term strategy in each search engine (Table 1), searching in article topics, titles, abstracts, and keywords. Where possible, filters were applied to retrieve articles in the English language and with human participants only. There was no restriction in the search period. Table of contents of the most common journals (determined using the interquartile rule for outliers) and the reference lists of any relevant review articles identified throughout the process which had misophonia in the title, were also screened. Searches were last updated in June 2018.

[INSERT TABLE 1 ABOUT HERE] 


\section{Study Selection}

Using an iterative approach, all abstracts were assessed independently by two researchers as meeting the review criteria or not. Records were first screened by title and abstract and in the next stage by full text. There were no disagreements between authors regarding the inclusion or exclusion of a record for this review.

\section{Data extraction}

A data extraction form was created in Excel and was piloted by two reviewers on two records before finalising. Data were then extracted independently by two reviewers (IP and DH) according to the following items: First Author; Year of Publication; Title of study; Publication Type; Publication type: other; Country study aim; Misophonia definition; Study design; Methods; If combination methods we specified; Sample size; percentage of participants with misophonia; Study population ; Setting; Age; Gender; Misophonia primary or secondary complaint; Age at onset; Trigger sounds; Response to the trigger sounds; Aggressive reaction use menu; Coping mechanisms; Other co-morbidities; Assessment time points; Eligibility screening methods; Assessments/ Outcome measures; Treatment aimed primarily at misophonia; Nature of therapeutic intervention (components); Main outcomes of study - empirical; Main outcomes of study - anecdotal; Study limitations; Further research; Notes. Disagreement on the extracted data was minimal and generally related to omissions rather than errors. Where these occurred both authors revisited the record to agree a final dataset. The final datasets were then charted by topic according to the aims of the review, namely the definition, patient profile, assessment (diagnosis and measurement), and treatment of misophonia. We additionally catalogued research priorities nominated in the literature.

\section{Results}


Figure 1 displays the flow of records identified, screened, included, or excluded, with reasons for exclusion. Thirty-one records were included in this review; 17 were case studies or case series, six were non-randomized controlled trials, two were online surveys and six were cohort studies. The earliest study was published in 2008 and one third of them were published in 2017. Fourteen studies were conducted in the US, eight in the Netherlands, two in Australia, two in the UK, two in Brazil, one in China, one in Turkey, and one in Canada. Six studies included children (aged under 18 years old) only, 17 studies included adults only, and six studies had a mixed population. Two studies could not be classified according to age as only mean sample ages were reported. Further study details are available in Supplementary materials 1 and 2 .

\section{[INSERT FIGURE 1 ABOUT HERE]}

\section{Definitions of misophonia}

Common terminology was observed in most definitions of misophonia used in the literature. Most commonly it was described as a disproportionately extreme, aversive, and irrational emotional reaction (Bernstein et al., 2013; Boyce 2015; Colucci 2015; Dozier 2015a,b; Edelstein et al., 2013; Kumar et al., 2014; Webber et al., 2014; Kumar et al., 2017; Erfanian, Brout \& Keshavarz. 2017, Tunç \& Başbuğ 2017; Vidal et al., 2017; Schneider \& Arch 2017; McKay et al., 2017; Sanchez \& de Silva 2017; Rouw \& Erfanian 2017) to specific (Boyce 2015, Colucci 2015, Edelstein et al., 2013, Giorgi et al., 2015; Webber et al., 2014; Reid et al., 2016; Wu et al., 2014; Tunç \& Başbuğ 2017; Schröder, Vulink, van Loon \& Denys 2017; Vidal, Vidal, \& Lage 2017; Schneider \& Arch 2017; McKay, Kim, Mancusi, Storch \& Spankovich 2017; Sanchez \& de Silva 2017; Rouw \& Erfanian 2017) common human innocuous sounds (Dozier 2015b,c; Johnson et al., 2013; Schröder et al., 2013a; Schröder, 
San Giorgi, Van Wingen, Vulink \& Denys 2015; Kumar et al; 2017, Schröder et al., 2017; Schneider \& Arch.,2017) and sometimes animal sounds (Schneider \& Arch 2017). Some authors described in detail the eliciting stimuli of this reaction. Two authors mentioned in their definitions the term 'trigger sounds' (Dozier 2015a; Webber et al., 2014) and some explained what these were exactly: breathing, wheezing, chewing, eating, slurping, lip smacking, pen clicking/tapping, typing, or cracking knuckles (Schröder et al., 2013a;2014; 2015; 2017; Kumar et al., 2017; Schneider \& Arch 2017; Sanchez \& de Silva 2017; McKay et al., 2017). Two authors (Hadjipavlou et al., 2008; Wu et al., 2014) described misophonia as a condition of decreased sound tolerance and six authors mentioned selective sound sensitivity syndrome (4S) as a synonym of misophonia (Kluckow, Telfer \& Abraham 2014; Webber et al., 2014; McGuire et al., 2015; Wu et al., 2014; Sanchez \& de Silva 2017; Zhou, Wu \& Storch 2017). The literal meaning of misophonia, hatred of sound, was used in the definition provided by some authors. (Edelstein et al., 2013; Kumar et al., 2014; Giorgi 2015; Schröder et al., 2013b; Wu et al., 2014).

Most authors described emotional reactivity, commonly experienced by patients, as part of their working definition. It is reported that emotional reactions "range or escalate" (Dozier 2015a) from discomfort (Wu et al., 2014), anxiety, distress (Johnson et al., 2013; Wu et al., 2014) to irritation, disgust, hate, anger, and loss of self-control (Boyce 2015; Colucci 2015, Dozier 2015a,b,c; Kumar et al., 2014; Giorgi 2015; Schröder et al., 2013a; Schröder et al., 2015; Webber et al., 2014, Reid et al., 2016; Wu et al., 2014; Kumar et al., 2017; Schröder et al., 2017). Some authors also referred to aggressive outbursts as a central hallmark of the condition (Schröder et al., 2013a; Schröder et al., 2014; Webber et al., 2014; McGuire et al., 2015; Kluckow et al., 2014; Tunç \& Başbuğ 2017). In addition to an emotional reaction, some authors referred to physiological or autonomic arousal (in terms of a trigger) in their 
definitions of misophonia (Bernstein et al., 2013; Edelstein et al., 2013; Vidal et al., 2017). Some definitions also noted the presence of coping mechanisms, typically avoidance strategies (Kumar et al., 2014; Schröder et al., 2015; Webber et al., 2014; McGuire et al., 2015; McKay et al., 2017).

\section{The misophonia population}

Onset

Almost all studies which reported onset age of misophonia found that symptoms emerged in childhood (Dozier 2015a,b,c; Johnson et al., 2013) or adolescence (i.e. up to 18 years of age) (Hadjipavlou et al.,2008; Kumar et al., 2014; Bernstein et al., 2013; Johnson et al., 2013; Kluckow et al., 2014; McGuire et al., 2015; Reid et al.,2016; Schröder et al., 2013b; Kamody \& Del Conte 2017; Schneider \& Arch 2017). Two studies reported that the onset was in adulthood (i.e. 18 years and over) (Boyce 2015; Tunç \& Başbuğ 2017) and two studies reported an onset age that can spread over the lifespan (Sanchez \& de Silva 2017; Zhou et al., 2017).

\section{Triggers and context}

Person-related sounds reported to trigger misophonic responses included oral sounds, especially those made by people eating, such as chewing, lip smacking, swallowing noises, and throat noises (Boyce 2015; Colucci 2015; Dozier 2015b; Hadjipavlou et al., 2008; Kumar et al., 2014; Schröder et al., 2015; 2017; Bernstein et al., 2013; Dozier 2015a,c; Johnson et al., 2013; Reid et al., 2016; Schröder et al., 2013b; Schröder et al., 2014; Wu et al., 2014; Kumar et al., 2017; Vidal et al; 2017; Schneider \& Arch 2017; Sanchez \& de Silva 2017; Zhou et al., 2017; Tunç \& Başbuğ 2017; Kamody \& Del Conte 2017), nasal sounds, such as breathing, blowing nose, sniffling, coughing (Dozier 2015a; Schröder et al., 2015; Dozier 
2015c; Johnson et al., 2013; Schröder et al., 2013b; Schröder et al., 2014; Wu et al.,2014; Kumar et al., 2017; Sanchez et al., 2017; Zhou et al., 2017; Kamody \& Del Conte 2017), and in single cases, nail picking (Hadjipavlou et al., 2008) or sneezing (Dozier 2015c). Specific spoken sounds, such as /s/, /t/, /ch/, /k/ (Colucci 2015; Wu et al., 2014), or consonants and/or vowels (Zhou et al., 2017) and specific voices (Boyce 2015; Hadjipavlou et al., 2008; Webber et al., 2014) were also reported as triggers. The context in which the triggers are experienced also appears important. Authors observed that triggers experienced within a familiar context, i.e. a family, friends, and occupational, or educational setting were more likely to cause a stronger misophonia reaction (Dozier 2015b; Edelstein et al., 2013; Bernstein et al., 2013; Dozier 2015a,c; Johnson et al., 2013; Kluckow et al., 2014; McGuire et al., 2015; Boyce 2015; Edelstein et al., 2013; Schneider \& Arch 2017).

Other common trigger-sounds include repetitive clicking and tapping (Colucci 2015; Johnson et al., 2013; McGuire et al., 2015; Reid et al., 2016; Schröder et al., 2014; Wu et al., 2014; Vidal et al., 2017; Zhou et al., 2017), or sounds produced by using utensils, keys, or ice in a glass (Dozier 2015b, c; Johnson et al., 2013; Sanchez \& de Silva 2017). Individual cases also describe bird chirps (Dozier 2015b; Johnson et al., 2013), dog barking (Kluckow et al., 2014; Sanchez \& de Silva, 2017), rustling (Schröder et al., 2014; Sanchez \& de Silva 2017; Zhou et al., 2017), and conversations in Spanish in a Hispanic individual who spoke both Spanish and English, as triggers (McGuire et al., 2015).

In some cases (Dozier 2015a,b; Hadjipavlou et al., 2008; Schröder et al., 2014; Vidal et al., 2017), triggers included person-related visual stimuli accompanying the auditory stimuli such as swinging arms or legs, jiggling, or rubbing hands together, which can fall under the term misokinesia. 


\section{The misophonic reaction}

All authors describe the misophonic reactions of their participants as various combinations of discomfort, distress, anxiety, annoyance, disgust, panic, extreme irritation, anger, hate, or sometimes rage with aggressive outbursts or involuntary violence (Colucci 2015; Dozier 2015b, Edelstein et al., 2013; Hadjipavlou et al., 2008; Webber et al., 2014; Kumar et al., 2014; Bernstein et al., 2013; Schröder et al., 2013b, 2017; Dozier 2015a,c; Johnson et al., 2013; Kluckow et al., 2014; McGuire et al., 2015; Reid et al., 2016; Kumar et al., 2017;Vidal et al., 2017; Schneider and Arch 2017; Sanchez and de Silva 2017; Rouw \& Erfanian 2017; Tunç \& Başbuğ 2017; Kamody \& Del Conte 2017 ). A larger qualitative study based on 42 people with misophonia (Schröder et al., 2013b) reports that physical aggression was present as a reaction in five of them. A physical reaction to trigger sounds appeared common and included contraction, tension, or sensation in specific muscles (Dozier 2015a,b,c; Bernstein et al., 2013; Kluckow et al., 2014; Kamody \& Del Conte 2017); and/or heavy breathing (Kluckow et al., 2014; McGuire et al., 2015). Edelstein et al., (2013) stated that the most common reaction reported in their sample was pressure experienced in the chest, arms or head, muscle tension, or a 'fight or flight' reaction (i.e. an autonomic stress response). One single case was noted to experience intrusive images of mouths and urges to mimic this (Hadjipavlou et al., 2008); another described feelings of shame and remorse (Bernstein et al., 2013).

\section{Coping strategies}

Patients managed their symptoms through avoidance behaviour e.g. leaving the situation, wearing earphones, or avoiding situations where the trigger might occur including quitting work (Edelstein et al., 2013; Kumar et al., 2014; Webber et al. 2014; Boyce 2015; Colucci 
2015; Dozier 2015a,b,c; Hadjipavlou et al., 2008; Johnson et al., 2013; Kluckow et al., 2014; McGuire et al., 2015; Reid et al., 2016; Schröder et al., 2013b; Schneider and Arch 2017; Kamody \& Del Conte 2017). Other strategies included confrontation i.e. demanding the offender (e.g. family member) to stop producing the trigger sound (Dozier 2015a,b; Webber et al., 2014; Edelstein et al., 2013; Johnson et al., 2013; Kluckow et al., 2014; Reid et al., 2016; Sanchez and de Silva 2017); also mimicking the offensive sound (Edelstein et al., 2013; Hadjipavlou et al., 2008; Schröder et al., 2013b), engaging in a positive internal dialogue (Edelstein et al., 2013, Kluckow et al., 2014; Reid et al., 2016), or distracting themselves from the sound (Kluckow et al., 2014) by producing anti-sounds (Schröder et al., 2013b) or diverting their thoughts (Schröder et al., 2017). Two single cases reportedly managed their symptoms using muscle relaxation exercises (Dozier 2015b). One case restrained herself from aggressive verbal outbursts by keeping her mouth full of food at meal times (Kluckow et al., 2014).

\section{Comorbid conditions}

Misophonia was noted to be co-morbid with OCD (Webber et al., 2014; Johnson et al., 2013; Kluckow et al., 2014; Reid et al., 2016; Schröder et al., 2013b; 2017, Wu et al., 2014; Erfanian et al., 2017), depression (Dozier 2015a; Johnson et al., 2013; Reid et al., 2016; Wu et al., 2014; Schroder et al., 2017; Erfanian et al., 2017), attention deficit hyperactivity disorder (ADHD) (Reid et al., 2016; Schröder et al., 2013b; 2014; 2017), asthma (Dozier 2015a,b; Kluckow et al., 2014), Tourette Syndrome (Wu et al. 2014; Schröder et al., 2013b; 2017), dysthymic disorder (Schröder et al., 2017; Erfanian et al., 2017); eating disorders (Kluckow et al., 2014; Schröder et al., 2017), post-traumatic stress disorder (PTSD) (Dozier 2015b; Erfanian et al., 2017); social phobia (Hadjipavlou et al., 2008; Erfanian et al., 2017); 
body dysmorphic disorder (Hadjipavlou et al.,2008; Schröder et al., 2017) and panic disorder (Hadjipavlou et al., 2008; Erfanian et al.,2017).

Individual cases were also reported that had borderline personality disorder (Boyce 2015), tinnitus and hearing loss (Colucci 2015), specific phobia (Reid et al., 2016), agoraphobia (Erfanian et al., 2017), generalized anxiety disorder (Dozier 2015c), Kawasaki disease (Johnson et al., 2013), hypochondria (Schröder et al., 2013b), skin picking (Schröder et al., 2013b; 2017), and bipolar disorder (Schröder et al., 2013b; 2017). It is important to note that although these various conditions were present alongside misophonia there is no evidence as yet to suggest any association. A future study should examine the prevalence of these comorbid conditions to evaluate which, if any, are in fact more prevalent in people with misophonia.

\section{Neurobiological findings}

Six records included neurobiological data from patients with misophonia. Edelstein et al. (2013) and Kumar et al. (2017) measured skin conductance during exposure to a range of trigger sounds in people with misophonia and healthy controls. They found that those with misophonia assign higher aversion, distress, and annoyance ratings, and have higher skin conductance or galvanic skin responses to auditory but not visual stimuli.

Giorgi (2015) used fMRI to explore brain areas involved in the misophonia reaction. They found hyperactivation in the bilateral auditory cortex and the left amygdala in patients with misophonia when exposed to misophonia triggers but not when the stimuli were neutral. Another fMRI experiment by Schröder et al. (2015) also found increased activity in the auditory cortex and the left amygdala. Schröder et al. (2013a) measured the mismatch 
negativity response - an objective test of automatic central auditory processing, finding it to be reduced in patients with misophonia compared to controls outside of any audiological distortions. The most recent work in this area (Kumar et al., 2017) suggests that misophonia is directly associated with an abnormal function of the anterior insular cortex (AIC) and a network of regions responsible for processing and regulation of emotions including ventromedial prefrontal cortex, posteromedial cortex, hippocampus, and amygdala. Kumar et al. (2017) also found that triggers elicited heightened heart rate and galvanic skin response in misophonia subjects, which were mediated by AIC activity. Questionnaire data indicated that patients with misophonia had higher interoceptive sensibility than controls, consistent with abnormal functioning of anterior insular cortex. Brain structural measurements implied greater myelination within ventromedial prefrontal cortex (vmPFC) in misophonic individuals.

Schröder et al. (2014) used EEG and an oddball paradigm to measure auditory event-related potentials (ERPs) evoked by pure tones between patients with misophonia and control participants. They found that the mean amplitude of the auditory N1, a marker linked to early attention and detection of abrupt sensory changes, was significantly diminished in patients with misophonia. Notably though, their patient group exhibited a higher total mood disturbance score than the controls which, the authors suggest, may reflect a general state of hyperarousal in patients' emotional state.

\section{Prevalence}

Two studies provide some prevalence data on misophonia. Wu et al. (2014) reported that in their sample of 483 undergraduate students $20 \%$ were classed as having clinically significant misophonia symptoms that cause interference in their lives. Another study with a sample size 
of 415 also suggested a prevalence rate of $20 \%$ of 'often' or 'always' experiencing sound sensitivity to triggers. However, this rate dropped to $6 \%$ for significantly interfering misophonia symptoms (Zhou et al., 2013). Kluckow et al. (2014) reported that, in a sample of 15 patients with eating disorders three also had misophonia, as determined from the Amsterdam Misophonia Scale (A-Miso-S) (Schröder et al., 2013b) and the Misophonia Activation Scale (MAS-1) (Fitzmaurice 2010).

\section{Diagnosis and measurement}

Most studies used an unstructured clinical interview to assess misophonia (Dozier 2015b, Hadjipavlou et al., 2008, Bernstein et al., 2013; Reid et al., 2016, Boyce 2015, Edelstein et al., 2013; Kamody \& Del Conte 2014; Giorgi 2015; Webber et al., 2014; Johnson et al., 2013; Kluckow et al., 2014; Schröder et al., 2013b; 2017; Vidal et al; 2017; Schneider and Arch 2017; Sanchez and de Silva 2017). Three studies specified use of the structured clinical interview for DSM IV Axis I or Axis II to diagnose patients with misophonia (Bernstein et al., 2013; Schröder et al., 2013b; Tunç \& Başbuğ 2017), and one used the M.I.N.I.

International Neuropsychiatric Interview for the diagnosis of affective disorders (Erfanian et al., 2017).

Different questionnaire measures of misophonia severity were used across studies. The most commonly used was the Amsterdam Misophonia Scale (A-Miso-S; Schröder et al., 2013b) (Reid et al., 2016; Giorgi 2015; Schröder et al., 2015; 2017; Kluckow et al., 2014; Schröder et al., 2013b; Schröder et al., 2014; Schneider and Arch 2017; Rouw and Erfanian 2017; Tunç \& Başbuğ 2017; Kamody \& Del Conte 2017) which is based on the Yale Brown Obsessive Compulsive Scale (Y-BOCS). Dozier (2015a,b) used the Misophonia Assessment Questionnaire $(M A Q)$ and the Misophonia Coping Responses Survey $(M C R)$ which, 
anecdotally, were both proposed by audiologist Marsha Johnson, although no information is available to verify this or how the questionnaires were developed. Dozier $(2015 \mathrm{~b}, \mathrm{c})$ used the Misophonia Trigger Severity Scale (MTS) by Natan Bauman (no reference identified). Kluckow et al. (2014) and Dozier (2015b) used the Misophonia Activation Scale - MAS-1 (Fitzmaurice 2010). The Misophonia Physiological Scale (MPS) was used by Dozier 2015c and has been developed by Natan Bauman (no reference identified).

The most recently published questionnaires are the Misophonia Emotional Response (MER; Dozier et al., 2015 b, c), and the Misophonia Questionnaire (MQ; Wu et al., 2014) (also used in, McGuire et al., 2015; Dozier 2015b; McKay et al., 2017; Zhou et al., 2017). The Misophonia Severity Scale (MSS), a sub-scale of the MQ, was adapted from the NIMH Global Obsessive-Compulsive Scale (NIMH GOCS; Murphy, Pickar \& Alterman, 1982). The MSS was used in two other studies (Dozier 2015b; McGuire et al., 2015). None of the available questionnaires have been yet been subjected to formal psychometric evaluation.

\section{Treatments approaches for misophonia}

Twelve studies explored treatments for misophonia. Three studies investigated a CBT method (McGuire et al., 2015; Bernstein et al., 2013; Schröder et al., 2017). In one study, a CBT subtype called Exposure and Response Prevention (ERP) was documented for a patient with a primary complaint of OCD (Reid et al., 2016). Three studies investigated counterconditioning treatment (Dozier et al., 2015 a,b,c). One study used mindfulness and acceptance to treat misophonia (Schneider \& Arch, 2017) and one used Dialectical Behaviour Therapy (DBT) (Kamody \& Del Conte 2017). One study (Hadjipavlou et al., 2008) documented two cases treated with a combination of exposure therapy, relaxation, and medication. Three studies 
(McGuire et al., 2015; Vidal et al., 2017; Tunç \& Başbuğ 2017) reported the results of using medication alone to treat misophonia.

Bernstein et al., (2013) described a case where CBT treatment comprised of challenging dysfunctional automatic thoughts, and a behavioural component to interrupt and substitute maladaptive and avoidant coping strategies with helpful ones. These included redirecting attention and talking openly with people producing the triggers in a non-offensive manner. There was also a physiological component involving exercise before meals to recalibrate autonomic reactivity. At the end of treatment, the case still found chewing noises unpleasant, but the triggers no longer impaired her social or occupational functioning and this effect was maintained at a 4-month follow-up assessment.

In McGuire et al. (2015) CBT included psychoeducation and achieving "habituation" through a combination of exposure and cognitive restructuring. More specifically, the two cases were exposed to triggers titrated upward in frequency and intensity to habituate them to the distress caused by the triggers. The process was then switched from the therapist to family members. To restructure dysfunctional beliefs about the triggers, cognitive restructuring was practiced during the exposures. Relapse prevention strategies were also introduced towards the end of treatment. Both cases showed improvement in misophonia symptoms based on their scores on the MQ and MSS, and anecdotal patient reports.

Schröder et al. (2017) tested an adaptation of group CBT on 90 patients. Components included task concentration exercises to train patients to shift their attention to neutral stimuli during exposures; counterconditioning was also used to break the association patients had between trigger sounds and experiencing negative emotions. Stimulus manipulation was the 
third component in this therapy. Patients were taught how to assume a sense of control over their responses by manipulating the trigger sounds they exposed themselves to on a computer at home as part of homework. Relaxation exercises were also included to help patients manage feeling of anger and rage, on the assumption that misophonia patients' irritability is related to an increased general arousal. This version of therapy significantly reduced misophonia symptoms in nearly half of patients and was found especially useful for more severe cases of misophonia.

Reid et al. (2016) investigated a single patient who had misophonia and comorbid OCD, depression, and specific phobia. In this case only two of the 14 sessions of CBT Exposure Relapse Prevention (CBT ERP) treatment were aimed at managing the misophonia problem. CBT ERP involved working with the patient through imaginal and in-vivo exposures to the stimulus. The structure of the two sessions for misophonia were not specified in the study so it is unclear whether these two sessions used ERP to treat the misophonia symptoms or whether it was standard CBT. The two misophonia sessions occurred in the middle of treatment for OCD. The patient also completed two related homework sessions. At the end of treatment, this patient's misophonia reduced from severe to mild/subclinical based on her AMiso-S score, and this improvement was maintained at 3-month follow-up.

Schneider \& Arch (2017) applied mindfulness and acceptance methods to treat misophonia in one case. Components were derived from Acceptance and Commitment Therapy (ACT) (acceptance, mindfulness, diffusion, and values) and DBT (acceptance, mindfulness, “nonjudgmentalness", and opposite action) perspectives, although several techniques could also be conceptualized from a classic CBT perspective (e.g., identifying the link between thoughts, feelings, and behaviours). Treatment consisted of 10 x 50-min sessions. Specific 
techniques included diffusion exercises (being aware of one's thoughts to create distance between the patient and the thoughts rendering them less overwhelming); DBT-style chain analyses to compare situations eliciting stronger and weaker reactions. A component of removing judgement was also included where the patient practiced noticing his judgmental thoughts and re-describing the situation objectively (just the facts). This method was effective 2 months later, and 6 months post-treatment, whereby the patient's scores across all measures including the A-Miso-S and Y-BOCS continued to decrease. The patient was better able to accept and tolerate the discomfort triggered by his misophonia.

Dialectical Behaviour Therapy (DBT) was used in one case (Kamody \& Del Conte, 2017) suffering comorbid anxiety and misophonia. DBT sessions were weekly for 7 weeks. The essence of the treatment was to acquire DBT skills of mindfulness and distress tolerance; to understand one's ability to transact differently with the environment despite his/her predisposition to anger, find alternative strategies to dealing with anger and guilt, and reduce behavioural engagement in behavioural urges. During treatment the patient was also given paroxetine hydrochloride (an SSRI) of $25 \mathrm{mg}$ (no frequency given) to simultaneously manage misophonic and anxiety symptoms. The authors reported a symptom reduction from severe to mild based on the A-Miso-S.

"Exposure therapy" was investigated in two cases (Hadjipavlou et al., 2008). The authors did not give information as to what the "exposure therapy" constituted, or whether it was part of CBT. This kind of therapy had limited effects in both cases due to non-compliance and perceived lack of benefit. The authors reported that alongside "exposure therapy" the two cases also received medication and relaxation treatment - although it is unclear if it was simultaneous. In the first case, the selective serotonin reuptake inhibitors (fluoxetine; dose 
not specified) combined with relaxation techniques provided symptomatic relief. In the other case, a 25-year-old woman with multiple morbidities, antidepressants, anxiolytics, antipsychotics, stimulants, and mood stabilizers were all ineffective, as were sound generators. McGuire et al. (2015) reported that a regimen of venlafaxine (150mg/day) (a serotonin-norepinephrine reuptake inhibitor) and lisdexamfetamine (70mg/day) (a central nervous system stimulant) led to moderate improvement in misophonia symptoms. However, the case had still suffered significant functional impairment and subsequently received CBT treatment.

Counterconditioning is a treatment which involves the pairing of a powerful, positive stimulus with a conditioned trigger stimulus causing the conditioned reflex to cease or die out. This method was specifically adapted to treat misophonia, and was tested in three studies (Dozier 2015a,b,c). It involves multiple treatment sessions in which a low intensity, short duration trigger stimulus was intermittently provided during a continuous positive stimulus such as music. Patients were encouraged to practice muscle relaxation during the treatments and to complete homework sessions using appropriate devises. Two studies testing this methodology were case studies, and one was a case series. Anecdotal patient reports suggest some improvement in patient symptoms; no empirical data were provided.

Two studies used medication alone to treat misophonia. Vidal et al. (2017) report on a single case of misophonia and obsessional symptoms treated with escitalopram (SSRI) (no dose detail given). Evaluation after 30 days showed partial relief from misophonia symptoms. In Tunç \& Başbuğ (2017) a case was treated with alprazolam (a benzodiazepine) ( $0.5 \mathrm{mg} /$ day) for three days to which the patient had responded well although misophonia persisted. 


\section{Research priorities nominated in the literature}

Suggestions for future research on misophonia in the literature included the need to confirm the phenomenology and prevalence of misophonia (Hadjipavlou et al., 2008), and its genetic and neurological mechanisms (Edelstein et al., 2013; Hadjipavlou et al., 2008; Webber et al., 2014; Dozier 2015a; Schröder et al., 2014; Giorgi 2015: McKay et al., 2017; Tunç \& Başbuğ 2017). Edelstein et al. (2013) also suggests investigating the natural history of misophonia and the evolution of triggers over time. There are also suggestions for further research into hereditary factors in misophonia (Sanchez and de Silva, 2017; Rouw and Erfanian 2017) and its comorbidties (Erfanian et al., 2017). There appears to be a consensus that conducting RCTs of the available treatments for misophonia is urgent (Dozier 2015b,c; Edelstein et al., 2013; Hadjipavlou et al., 2008; Reid et al., 2016; Schröder et al., 2017; Schneider and Arch 2017; Kamody \& Del Conte 2017). Dozier (2015b) proposed that a set of validated assessment instruments for misophonia should be established. The effectiveness of CBT and how it links with brain responses was specifically suggested (Giorgi 2015; McGuire et al., 2015; Reid et al., 2016). Finally, investigating misophonia within certain populations (i.e. paediatric, autism spectrum disorder, eating disorders, and tinnitus) in order to garner a better clinical picture of misophonia, and to clarify its association with tinnitus, was mentioned by a number of authors (Wu et al., 2014; Kluckow et al., 2014; McGuire et al., 2015; Zhou et al., 2017).

\section{Discussion}

This scoping review catalogues the research describing misophonia, its population, methods of assessment, treatments, and future research priorities. Inherent to scoping review methodology, we included all the relevant and available research studies on misophonia treatments. We found that most studies used methodologies that provide low level evidence, 
with over half of the current research on misophonia taking the form of case studies. This finding highlights the need for controlled studies and clinical trials on misophonia.

Misophonia is a condition only described as such in the last two decades and no official diagnostic criteria have yet been formulated. Although Schröder et al. (2013b) proposed a set of diagnostic criteria, this has not been formalised. We scoped the available definitions of misophonia used by researchers, and found a high level of common terms and statements. Still some definitions were broad and did not seem to differentiate misophonia from hyperacusis. The collation of definitions presented here can be used in finding consensus on a single definition and description of misophonia. It also serves as a starting point to consider the validity of existing or new misophonia measurement questionnaires. These measures will, in turn allow more rigorous diagnostic profiling and evaluation of available treatments for misophonia.

Descriptions of the misophonia population consistently identified that its onset is in childhood or early adolescence at 12 or 13 years. There can also be individual cases where misophonia begins in childhood up or middle-age. As reported in a previous review (Bruxner et al., 2015) the triggers of the misophonia symptoms are almost always produced by humans, and most commonly involve oral and nasal sounds, with the level of familiarity of the context within which the triggers are experienced playing a significant role. Over the course of their condition some patients can develop intolerance to certain visual stimuli which accompany the audible ones, such as movement of mouths, and these can become triggers on their own. A few studies also report that repetitive noises such as clicking and tapping can also trigger misophonia symptoms. This suggests a possible continuum between misophonia and misokinesia (hatred of movement) (Schröder et al., 2013b). Misokinesia is 
not currently known to exist as a discrete condition but is rather a less common misophonia symptom.

Evidence on the prevalence of misophonia is only available from two student populations. Authors report a rate of $20 \%$ of people 'often' or 'always' experiencing sensitivity to triggers with $6 \%$ experiencing impairment. This could mean that while many may experience sound sensitivity to some triggers, a relatively low percentage suffer.

Few studies have been dedicated so far to studying the neurological correlates of misophonia. Of those conducted most used MRI or EEG and skin conductance. The findings are all considered preliminary but consistently suggest that misophonia involves abnormal functioning of the amygdala, an area of the brain commonly associated with negative emotions, aversive learning, and processes of attention and vigilance (Davidson \& Irwin 1999; Davis \& Whalen 2001; Holland \& Gallagher 1999). The most recent study reported here (Kumar et al., 2017) provides further evidence that it is the AIC and its connection to the amygdala, and other regions involved in emotion, that are malfunctioning in patients with misophonia. Currently it is still unclear whether the AIC malfunction is a cause or consequence of misophonia. The study found that patients also displayed a generally heightened baseline emotional state or hyperarousal, though there was evidence too that they had a lowered automatic central auditory processing mechanism. Further research is required to confirm these findings and how they link to the aetiology, progress, and maintenance of misophonia.

Some of the cases we reviewed could potentially be classed as being 'extreme' and not representative of the typical misophonia population. However the current evidence base on 
the symptomatology of misophonia cannot differentiate these for certain. On the whole we found that the misophonic reaction frequently reported in research involves intense negative feelings which can vary from anxiety, disgust, panic, discomfort, annoyance, frustration, and rage, with the latter reported as a key characteristic (Schröder et al., 2013; 2014; Dozier 2015a,b,c; Kumar et al., 2014). Patients may experience anxiety in anticipation of hearing the trigger sounds and then anger and disgust when they actually hear them. The literature we reviewed suggests that the dominant misophonia reaction involved intense negative feelings (Schröder et al., 2013b; 2014; Dozier 2015a,b,c; Kumar et al., 2014; 2017) but also anxiety or fear of being unable to control the intolerance (Edelstein 2013; Hadjipavlou et al., 2008; Kumar et al., 2014). Research also showed that the misophonic reaction is commonly associated with muscle tension in various body regions, symptoms related to anxiety (Sainsbury \& Gibson 1954; Ginsburg, Riddle \& Davies 2005), as well as abnormal physical stress (Rouw \& Erfanian 2017). In line with this, Wu et al. (2014) and Zhou et al. (2017) found that it is indeed anxiety which mediates the link between sound intolerance and anger outbursts. This idea is also supported by the model proposed by Webber and Storch (2015) highlighting the central role of anxiety which is negatively reinforced by behavioural responses, and sheds light on the treatment of misophonia. Therefore, it appears that although anger is the dominant misophonic reaction, anxiety caused by the anger, affects the consequent behavioural responses such as intrusive compulsions or safety behaviours. These behavioural consequences may lead to interpersonal and occupational dysfunction (Schwartz et al. 2011; Cavanna 2014) causing decreased quality of life which, in turn causes more anxiety. Further highlighting the role of anxiety in misophonia we found that the condition most commonly reported to be comorbid with misophonia was the anxiety disorder OCD. Schröder et al. (2013b) highlighted that both disorders share the intrusive and unwanted preoccupation and compulsive stress-reducing and avoidance behaviours. For this reason, he 
has proposed that misophonia could be classified within the obsessive compulsive spectrum of disorders of the DSM-IV. The parallels between misophonia and OCD also informed the development of the A-MISO-S and the MSS. However, the psychometric properties of these two measures, as well as other less commonly used outcome measures for misophonia, are yet to be established.

Evidence on effective treatments for misophonia is scarce. Less than half of the available studies on misophonia focused on treatment, and all but one were case studies. Hence in reviewing, one must be conscious of the potential 'file drawer effect', whereby only cases of effective treatment get published. The most researched type of treatment for misophonia was CBT in various adaptations, whereby achieving habituation to the triggers through in-vivo exposures, was the common component amongst them. These proved effective in all studies, as did counterconditioning (a type of behavioural treatment where controlled exposure to triggers to achieve habituation is also central but lacks the cognitive counselling element of CBT). Although these positive findings are based on anecdotal reports, there is a good foundation to conduct RCTs to ascertain the most effective format of CBT treatment. Other cognitive treatments such as mindfulness and acceptance and DBT also showed promising results and warrant further research. Certain medication, such as fluoxetine, venlafaxine, lisdexamfetamine, paroxetine hydrochloride, escitalopram, and alprazolam were used either in combination with a cognitive therapy or alone. Future research should compare and assess the exact effect of these drugs on misophonia. It is crucial to highlight that although such medication has been tested with misophonia patients this does not amount to evidence of effectiveness. RCTs are required to assess the safety dosages and whether or not they are effective. Currently only one clinical trial on the neural and self-reported effect of CBT has been identified as ongoing (ISRCTN:12571777). 


\section{Limitations}

Firstly, although this is a review of misophonia research, the definitions of misophonia used in the included records are varied and so the participants and patients described may not represent a homogenous population. Secondly, although we undertook the optional Stage 6 (consultation) of scoping reviews (Levac et al., 2010), there is a current lack of guidance on how this is best conducted. We chose to incorporate the clinicians' interpretations of the results from their experience within the discussion section. A more systematic process of conducting and reporting the consultation stage could enhance the value of this stage of the scoping review process.

\section{Conclusion}

Although no formal nosology of misophonia has yet been finalized, research to date shows consistency in terms of the characteristics of misophonia that are described. To date, there are no studies that provide a reliable estimate of the prevalence of misophonia in the general population, nor in specific patient populations. Demographic data would inform the true scale of the disorder and better define the scale of need for healthcare. Nevertheless research in misophonia has progressed in the last decade in that outcome measures of misophonia have been developed and some possible treatments have been shown effective in providing relief in case studies. Authors in the field agree the need for studies that validate existing outcome measures, RCTs of treatments, and studies to determine the aetiology and natural history of misophonia. The current evidence base on misophonia is not yet substantial enough to consider a systematic review. However, we suggest that RCTs testing possible treatments for misophonia is a vital next step for patients. Epidemiological research and consensus-based research is necessary for demographic estimations and to ascertain diagnostic criteria. 


\section{Acknowledgements}

IP and DJH are funded through the National Institute for Health Research (NIHR)

Biomedical Research Centre programme. The opinions expressed are those of the authors and not necessarily those of the NIHR, the NHS, or the Department of Health and Social Care.

\section{References}

Arksey, H., \& O'Malley, L. (2005). Scoping studies: towards a methodological framework. International Journal of Social Research Methodology, 8(1), 19-32.

Aromataris, E., \& Riitano, D. (2014). Systematic reviews: constructing a search strategy and searching for evidence. AJN The American Journal of Nursing, 114(5), 49-56.

Bernstein, R. E., Angell, K. L., \& Dehle, C. M. (2013). A brief course of cognitive behavioural therapy for the treatment of Misophonia: a case example. The Cognitive Behaviour Therapist, 6, e10.

Boyce, P. M. (2015). A young woman with noise intolerance. Medicine Today, 16(7): 46-47.

Bruxner, G. (2016). 'Mastication rage': a review of misophonia - an under-recognized symptom of psychiatric relevance. Australian Psychiatry, 24:2, 195 - 197.

doi: $10.1177 / 1039856215613010$

Cavanna, A. E. (2014). What is Misophonia and how can we treat it? Neurotherapeutics, 14(4), 357-359.

Cavanna, A. E., \& Seri, S. (2015). Misophonia: current perspectives. Neuropsychiatric disease and treatment, 11, 2117.

Colucci, D. A. (2015). A Case of Amplified Misophonia? The Hearing Journal, 68(2), 40.

Davis, M., \& Whalen, P. J. (2001). The amygdala: vigilance and emotion. Molecular Psychiatry, 6(1), 13.

Davis, K., Drey, N., \& Gould, D. (2009). What are scoping studies? A review of the nursing literature. International Journal of Nursing Studies, 46(10), 1386-1400.

Davidson, R. J., \& Irwin, W. (1999). The functional neuroanatomy of emotion and affective style. Trends in cognitive sciences, 3(1), 11-21.

Dozier, T. H. (2015a). Counterconditioning treatment for Misophonia. Clinical Case Studies, 14(5), 374-387.

Dozier, T. H. (2015b). Treating the initial physical reflex of Misophonia with the neural repattering technique. Psychological Thought, 8(2), 189-210. 
Dozier, T. H. (2015c). Etiology, composition, development and maintenance of Misophonia: A conditioned aversive reflex disorder. Psychological Thought, 8(1), 114-129.

Duddy, D. F., \& Oeding, K. A. (2014, May). Misophonia: An Overview. In Seminars in Hearing (Vol. 35, No. 02, pp. 084-091). Thieme Medical Publishers.

Eddy, C. M., \& Cavanna, A. E. (2014). Premonitory urges in adults with complicated and uncomplicated Tourette syndrome. Behaviour Modification, 38(2), 264-275.

Edelstein, M., Brang, D., Rouw, R., \& Ramachandran, V. S. (2013). Misophonia: physiological investigations and case descriptions. Frontiers in Human Neuroscience, 7, 296.

Erfanian, M., Brout, J. J., \& Keshavarz, A. (2017). Misophonia and affective disorders: The relationship and clinical perspective. European Psychiatry, 41, S471.

Fackrell, K., Potgieter I., Shekhawat, G. S., Baguley, D. M., Sereda, M. and Hoare D. J. (2017). Clinical Interventions for Hyperacusis in Adults: A Scoping Review to Assess the Current Position and Determine Priorities for Research. BioMed Research International, 2017.

Ferreira, G. M., Harrison, B. J., \& Fontenelle, L. F. (2013). Hatred of sounds: Misophonic disorder or just an underreported psychiatric symptom? Annals of Clinical Psychiatry, 25(4), 271-274.

Fitzmaurice, G. (2010). Misophonia activation scale (MAS-1). Available from:

http://www.misophonia-uk.org/the-misophonia-activation-scale.html. Accessed: 23.03.2017

Formby, C., Sherlock, L. P., \& Gold, S. L. (2003). Adaptive plasticity of loudness induced by chronic attenuation and enhancement of the acoustic background. Journal of the Acoustical Society of America, 114, 55-58.

Giorgi, R. S. (2015). Hyperactivity in amygdala and auditory cortex in Misophonia: preliminary results of a functional magnetic resonance imaging study. Amsterdam Brain and Cognition Journal, issue 2, p221.

Ginsburg G. S., Riddle M. A. and Davies M. (2005). Somatic symptoms in children and adolescents with anxiety disorders. Journal of the American Academy of Child and Adolescent Psychiatry. 45(10), 1179-1187.

Goodman, W. K., Price, L. H., Rasmussen, S. A., Mazure, C., Delgado, P., Heninger, G. R., \& Charney, D. S. (1989). The Yale-Brown obsessive compulsive scale: II. Validity. Archives of General Psychiatry, 46(11), 1012-1016.

Hadjipavlou, G., Baer, S., Lau, A., \& Howard, A. (2008). Selective sound intolerance and emotional distress: what every clinician should hear. Psychosomatic Medicine, 70(6), 739740 . 
Hazen, E. P., Reichert, E. L., Piacentini, J. C., Miguel, E. C., Do Rosario, M. C., Pauls, D., \& Geller, D. A. (2008). Case series: sensory intolerance as a primary symptom of paediatric OCD. Annals of Clinical Psychiatry, 20(4), 199-203.

Holland, P. C., \& Gallagher, M. (1999). Amygdala circuitry in attentional and representational processes. Trends in Cognitive Sciences, 3(2), 65-73.

Jastreboff, P. J., \& Jastreboff, M. M. (2013). Using TRT to treat hyperacusis, misophonia and phonophobia. ENT Audiol News, 21(6), 88-90.

Jastreboff, P. J., \& Hazell, J. W. (2008). Tinnitus retraining therapy: implementing the neurophysiological model. Cambridge University Press.

Jastreboff, P. J., \& Jastreboff, M. M. (2003). Tinnitus retraining therapy for patients with tinnitus and decreased sound tolerance. Otolaryngologic Clinics of North America, 36(2), 321-336.

Jastreboff, P. J., \& Jastreboff, M. M. (2014). Treatment for decreased sound tolerance (hyperacusis and misophonia). Seminars in Hearing, 35(2),105-120.

Jastreboff, M.M., \&Jastreboff, P.J. (2002). Decreased sound tolerance and Tinnitus Retraining Therapy (TRT). Australian and New Zealand Journal of Audiology. 24(2), 74-81.

Jastreboff, M. M., \& Jastreboff, P. J. (2001a). Components of decreased sound tolerance: hyperacusis, misophonia, phonophobia. ITHS News Lett, 2(5-7).

Jastreboff, M. M., \& Jastreboff, P. J. (2001b). Hyperacusis. Audiology Online. June 2001

Jastreboff, P. J. (2000). Tinnitus habituation therapy (THT) and tinnitus retraining therapy (TRT). Tinnitus Handbook, 357-376.

Johnson, P. L., Webber, T. A., Wu, M. S., Lewin, A. B., Murphy, T. K., \& Storch, E. A. (2013). When selective audiovisual stimuli become unbearable: a case series on pediatric misophonia. Neuropsychiatry, 3(6), 569-575.

Kamody, R. C., \& Del Conte, G. C. (2017). Using Dialectical Behavior Therapy to Treat Misophonia in Adolescence. The primary care companion for CNS disorders, 19(5).

Khalil, H., Peters, M., Godfrey, C. M., McInerney, P., Soares, C. B., \& Parker, D. (2016). An Evidence-Based Approach to Scoping Reviews. Worldviews on Evidence-Based Nursing, 13(2), 118-123.

Kluckow, H., Telfer, J., \& Abraham, S. (2014). Should we screen for misophonia in patients with eating disorders? A report of three cases. International Journal of Eating Disorders, $47(5), 558-561$.

Kumar, S., Tansley-Hancock, O., Sedley, W., Winston, J. S., Callaghan, M. F., Allen, M., ... \& Griffiths, T. D. (2017). The Brain Basis for Misophonia. Current Biology, 27, 1-7. 
Kumar, S., Hancock, O., Cope, T., Sedley, W., Winston, J., \& Griffiths, T. D. (2014). Misophonia: A disorder of emotion processing of sounds. Journal of Neurology, Neurosurgery \& Psychiatry, 85(8), e3-e3

Levac, D., Colquhoun, H., \& O'Brien, K. K. (2010). Scoping studies: advancing the methodology. Implementation Science, 5(1), 69.

Martz, K. (2013). Patient Panel. Presentation at the annual conference of the Misophonia Association, Portland, OR.

McFerran, D. (2016). "Misophonia and phonophobia." Baguley, David M [Ed]; Fagelson, Marc [Ed] (2016) Tinnitus: Clinical and Research Perspectives (pp 245-259) xi, 364 pp San Diego, CA, US: Plural Publishing; US: 245-259.

McGuire, J. F., Wu, M. S., \& Storch, E. A. (2015). Cognitive-behavioral therapy for 2 youths with misophonia. The Journal of Clinical Psychiatry, 76(5), 573-574.

McKay, D., Kim, S. K., Mancusi, L., Storch, E. A., \& Spankovich, C. (2017). Profile Analysis of Psychological Symptoms Associated With Misophonia: A Community Sample. Behaviour Therapy.

Møller, A. R. (2011). Misophonia, phonophobia, and "exploding head" syndrome. In Textbook of tinnitus (pp. 25-27). Springer New York.

Murphy, D. L., Pickar, D., \& Alterman, I. S. (1982). Methods for the quantitative assessment of depressive and manic behavior. In The behaviour of psychiatric patients (pp. 355-392). Marcel Dekker, New York.

Neal, M., \& Cavanna, A. E. (2013). Selective sound sensitivity syndrome (misophonia) in a patient with Tourette syndrome. The Journal of Neuropsychiatry and Clinical Neurosciences, 25(1), E01-E01.

Peters, M. D., Godfrey, C. M., Khalil, H., McInerney, P., Parker, D., \& Soares, C. B. (2015). Guidance for conducting systematic scoping reviews. International Journal of EvidenceBased Healthcare, 13(3), 141-146.

Reid, A. M., Guzick, A. G., Gernand, A., \& Olsen, B. (2016). Intensive cognitive-behavioral therapy for comorbid misophonic and obsessive-compulsive symptoms: A systematic case study. Journal of Obsessive-Compulsive and Related Disorders, 10, 1-9.

Rouw, R., \& Erfanian, M. (2017). A Large-Scale Study of Misophonia. Journal of Clinical Psychology.

Rumrill, P. D., Fitzgerald, S. M., \& Merchant, W. R. (2009). Using scoping literature reviews as a means of understanding and interpreting existing literature. Work (Reading, Mass.), 35(3), 399-404.

Sainsbury P. and Gibson J. G. (1954). Symptoms of anxiety and tension and the accompanying physiological changes in the muscular system. Journal of Neurology, Neurosurgery and Psychiatry, 17, 216. 
Sanchez, T. G., \& da Silva, F. E. (2017). Familial misophonia or Selective Sound Sensitivity Syndrome: evidence for autosomal dominant inheritance? Brazilian journal of

Otorhinolaryngology.

Schwartz, P., Leyendecker, J., \& Conlon, M. (2011). Hyperacusis and misophonia: the lesserknown siblings of tinnitus. Minnesota Medicine, 94(11), 42-43.

Schröder, A. E., Mahazeri, A., Petropoulos,D., Soto, V., Smolders, Z. Vulink, N .C.C., \& Denys, D. (2013a). A diminished mismatch negativity response in misophonia, a potential marker for aggressive impulsivity. European Neuropsychopharmacology, 23, S177.

Schröder, A., Vulink, N., \& Denys, D. (2013)b. Misophonia: diagnostic criteria for a new psychiatric disorder. PLoS One, 8(1), e54706.

Schröder, A., van Diepen, R., Mazaheri, A., Petropoulos-Petalas, D., de Amesti, V. S., Vulink, N., \& Denys, D. (2014). Diminished n1 auditory evoked potentials to oddball stimuli in misophonia patients. Frontiers in Behavioural Neuroscience, 8.

Schröder, A., San Giorgi, R., Van Wingen, G., Vulink, N., \& Denys, D. (2015). P. 1. i. 015 Impulsive aggression in misophonia: results from a functional magnetic resonance imaging study. European Neuropsychopharmacology, (25), S307-S308.

Schröder, A. E., Vulink, N. C., van Loon, A. J., \& Denys, D. A. (2017). Cognitive behavioral therapy is effective in misophonia: An open trial. Journal of Affective Disorders, 217, 289294.

Schneider, R. L. and Arch, J. J. (2017). Case study: a novel application of mindfulness- and acceptance-based components to treat misophonia. Journal of Contextual Behavioral Science, $6,221-225$.

Taylor, S., Conelea, C. A., McKay, D., Crowe, K. B., \& Abramowitz, J. S. (2014). Sensory intolerance: latent structure and psychopathologic correlates. Comprehensive Psychiatry, 55(5), 1279-1284.

Tricco, A. C., Lillie, E., Zarin, W., O’Brien, K., Colquhoun, H., Kastner, M., ... \& Kenny, M. (2016). A scoping review on the conduct and reporting of scoping reviews. BMC Medical Research Methodology, 16(1), 15.

Tricco, A.C., Lillie, E., Zarin, W., O'Brien, K.K., Colquhoun, H., Levac, D., Moher, D., Peters, M.D., Horsley, T., Weeks, L. and Hempel, S., 2018. PRISMA extension for scoping reviews (PRISMA-ScR): checklist and explanation. Annals of internal medicine, 169(7), 467473.

Tunç, S., \& Başbuğ, H. S. (2017). An extreme physical reaction in misophonia: stop smacking your mouth! Psychiatry and Clinical Psychopharmacology, 27(4), 416-418.

Vidal, C., Vidal, L. M., \& Lage, M. A. (2017). Misophonia: Case report. European Psychiatry, 41, S644. 
Webber, T. A., Johnson, P. L., \& Storch, E. A. (2014). Pediatric misophonia with comorbid obsessive-compulsive spectrum disorders. General Hospital Psychiatry, 36(2), 231-e1.

Webber, T. A., \& Storch, E. A. (2015). Toward a theoretical model of misophonia. General Hospital Psychiatry, 37(4), 369.

Wu, M. S., Lewin, A. B., Murphy, T. K., \& Storch, E. A. (2014). Misophonia: incidence, phenomenology, and clinical correlates in an undergraduate student sample. Journal of Clinical Psychology, 70(10), 994-1007.

Zhou, X., Wu, M. S., \& Storch, E. A. (2017). Misophonia symptoms among Chinese university students: Incidence, associated impairment, and clinical correlates. Journal of Obsessive-Compulsive and Related Disorders, 14, 7-12.

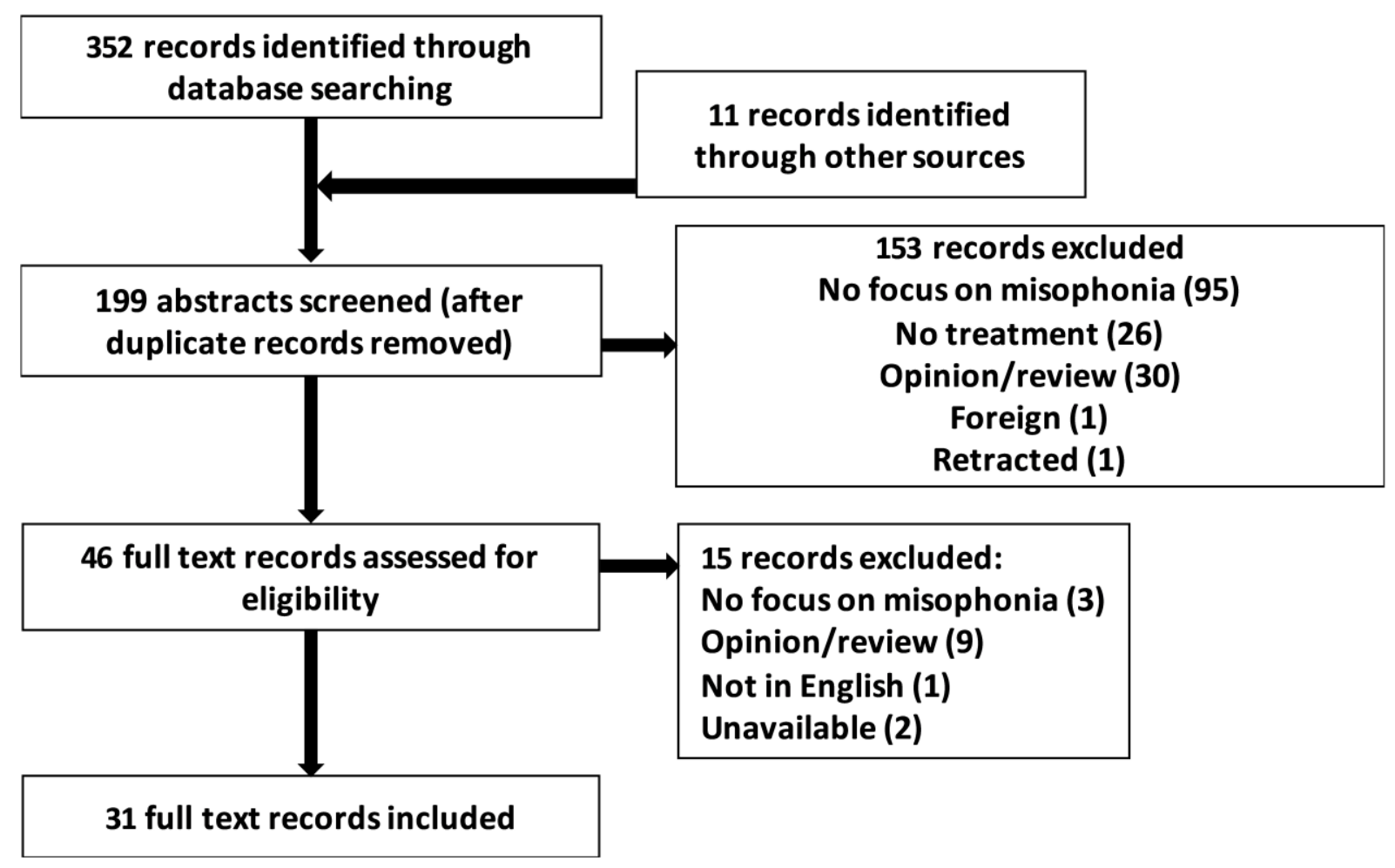

